\title{
ROBOTIC SURGERY - A STEP INTO THE FUTURE
}

\author{
Cirurgia robótica - um passo em direção ao futuro \\ Carlos Eduardo DOMENE \\ TCBCB and 1st Vice-President of Brazilian Society of Videosurgery - SOBRACIL
}

Laparoscopic surgery has been widely diffused since 1990, having exponential growth since then. Contributed to this fact the obvious benefits of the avoidance of large abdominal incisions, less surgical trauma, less perioperative morbidity, smoother and faster postoperative recovery, wide acceptance by patients, who started demanding technology. In addition, many surgeons quickly joined the laparoscopy and there was a great cooperative effort worldwide. Unprecedented and widespread dissemination of knowledge occurred, especially because the view on a monitor and its easy recording and retransmission, unlike open surgery everyone could see the proceedings, or repeatedly on videos.

Robotic surgery has been introduced from the year 2000 and has had marked increase in its use since them. The operations that are performed laparoscopically can be made through the robot, with more accuracy and safety. The use of robot favors a less invasive operation; with a much better view of the organs being operated; with great approximation of the structures; with the surgeon's vision in three dimensions; procedure even less invasive; and with less tissue trauma.

Who controls the movements of the robot is the surgeon through a special console, dominating every movement of the grippers and the camera. The clamps have more delicate movements, being literally controlled with fingertips.

It's possible to have great accuracy, due to the interface of the "robot" between the arms of the surgeon and the patient's operated organs. Robotic grippers are specially designed to simulate the movements of the surgeon's hands, allowing dexterity never achieved by laparoscopic surgery. The surgeon do not use any force to control the robotic arms, doing movements with the extremities of the fingers; thus, there is much less fatigue in prolonged procedures. The robot heps the trained surgeon perform operations even more safe and accurate.

Another relevant feature of the robot, of great importance is the possibility of extensive training in simulators. There is an outfit named MIMIC with programs that simulate situations of object manipulation, movement, energy use, sutures, etc. The surgeon is aware of their performance by an assessment that appears immediately after exercise, showing numerous variables that exercise demand, directing the aspect that need to be improved, or if it was correctly done. The surgeon can thus become familiar with the equipment and thorough training, perform initial procedures with more skill and accuracy, reducing the learning curve (as demonstrated in controlled studies) and possibly reducing the risk of occurrence of accidents and complications, which occur in the learning curve of surgery, either open, laparoscopic or robotic approach.

Robotic prostatectomy has been achieving similar oncological outcomes to laparotomy, with the advantage of better preservation of erectile function due to the precise dissection of the pelvic nerves. It is now considered a standard of excellence in the operation of prostate cancer. Robotic gynecologic surgery got significant increase in recent years, also leading to very good results.

In the digestive tract, virtually all operations can be performed through the assistance of the robot. In obesity surgery allows better access to organs, maximized visualization and high precision in the sutures. In esophageal surgery provides precise, anatomic, minor assault procedures. When operating the intestine, the robot must allow release of the structures, preserving vessels and nerves which help to preserve continence and potency functions, important to patients. Assisted by the robot operations greatly help the surgeon to bring greater benefit and safety for their patients, especially when there are anastomoses or dissections requiring high precision and privileged view; reoperations or revisions are thus much better performed with the aid of the robot. The dual console allows second surgeon to assist or interfere, facilitating training during the learning curve.

However, the most important point of this new technology is the introdution of a new paradigm in surgery: the existence of a device (called robot, in lack of a better name) that allows the use of computer programs for performing tasks. No other surgical platform, at present, has this feature. Laparoscopic forceps are directly controlled by the surgeon's hands and, with the exception of the power instruments, little change occurred over the past 20 years.

On the other hand, are well known the incredible advances in diagnostic medicine since the introduction of computer programs in imaging and interventional equipment; innovations in these areas occur almost daily. This same feature is now available to the surgeon. Computer programs can, for instance, be inserted to allow different examinations during operation; identification of lymph nodes compromised by tumor; differentiation over vessels, nerves and other tissues. From this premise, the possibilities are almost endless bring new technologies in the future.

The technology using green dye - binds to blood proteins - injected by peripheral vein is commercially available. Through fluoroscopy in a special camera the surgeon may identify differences between healthy and tumor cells; check the vascular supply of the parenchyma or viscera; identify the bile duct during cholecystectomy. The surgeon can quickly change from normal to fluoroscopic camera. Allows more accurate removal of tumors; make safer intestinal anastomosis for making sure the proper irrigation of the extremities; increase the security of difficult cholecystectomy, where the anatomy is often inaccurate or anomalous. It has been said that, as this technology allows for much better accuracy in the identification of the structures of the hepatic hilum and reduces the risk of inadvertent bile duct injury, it could be considered unethical not to offer this technology to patients.

The robotic platform is evolving exponentially. There is, at present, only one company with equipment released by the authorities for human use. There is already a new generation released for use next year, and new prototype is currently being tested. When released some patents, several new equipment, already in testing, will be available on the market. Then, it is possible to have important decrease in costs, which is now the main obstacle to its widespread use.

The robots are here to stay. The possibilities of computer programs interact are almost endless. Costs will decrease considerably in the next years. So, the future has arrived! 